\title{
Dynamic response of a vehicle with flexible car body moving on a ballasted track
}

\author{
Yingjie Wang ${ }^{1,2^{*}}$, Zuzana Dimitrovová ${ }^{3,4}$, and Jong-Dar Yau ${ }^{5}$ \\ ${ }^{1}$ School of Civil Engineering, Beijing Jiaotong University, Beijing, China. \\ ${ }^{2}$ Beijing Engineering and Technology Research Center of Rail Transit Line Safety and Disaster \\ Prevention, Beijing, China. \\ ${ }^{3}$ Department of Civil Engineering, Faculdade de Ciências e Tecnologia, Universidade Nova de \\ Lisboa, Caparica, Portugal. \\ ${ }^{4}$ IDMEC, Instituto Superior Técnico, Universidade de Lisboa, Lisboa, Portugal. \\ ${ }^{5}$ Department of Architecture, Tamkang University, New Taipei City, Taiwan.
}

\begin{abstract}
In this study, the dynamic response of a vehicle with flexible car body moving on a ballasted track is investigated. The vehicle/track interaction model is divided into two sub-systems, namely the vehicle and the ballasted track sub-system. The vehicle sub-system is composed by one flexible car body, two bogies and four wheel sets. The car body is created using flexible multibody dynamics. The ballasted track consists of rail, sleeper, ballast and sub-grade. For its representation the simplified 2D discrete support model (DSM) is used and modelled by finite elements. The connection between the two sub-systems, i.e. between the moving wheels and the track is ensured by a linearized Hertzian spring. The dynamic response of the vehicle/track system is computed by the Newmark- $\beta$ integration method. The effect of the car body flexibility on the dynamic response of this system is analyzed and evaluated based on the maximum car center acceleration. It is concluded that the increase in flexural stiffness of the car body can lead to a notable decrease of its maximum acceleration.
\end{abstract}

\section{Introduction}

The riding comfort of rail vehicles attracts significant attention of the academy and industry $[1,2]$. When the rail vehicle moves on a track structure, its vibrations are affected by both, the vehicle condition and the track condition. This concerns the vehicle suspensions, rail profile and track irregularities. To study the vibrations of the vehicle/track system, some researchers developed several kinds of vehicle-track models. Nguyen et. al [3] compared the dynamic response of the coupled vehicle-track system simplified by $2 \mathrm{D}$ finite element model and modelled by full 3D multibody/finite-element model and concluded that the 2D model can be used to predict the vertical dynamic response with sufficient accuracy. The 2D model in form of discrete elements connected on top by the rail, named as DSM, was further investigated in [4], where analysis of its applicability and general formulas for

\footnotetext{
* Corresponding author: ceyjwang@bjtu.edu.cn
} 
determination of its properties are derived. In this study, only moving forces were applied for simplicity. Varandas et. al [5] developed a 3D FEM dynamic program for the coupled train-track interaction analysis and studied the culvert influence on the train-track responses. In [3,5] and other similar works, the components of the vehicle model are regarded as rigid, and the flexible vibration of the car body is neglected.

In this paper, the dynamic response of a vehicle with flexible car body moving on a typical ballasted track is investigated. The vehicle is developed using rigid-flexible coupling dynamics and the track is simplified by DSM and modeled using finite elements. A connection between the two sub-systems is ensured by a linearized Hertzian spring accounting for the interaction between the moving wheel sets and the rail. The dynamic response of the vehicle/track system is computed by the Newmark- $\beta$ integration method. The dynamic response of the vehicle and ballasted track is compared in situations in which the car body is assumed as flexible and as rigid. At last, the influence of flexural stiffness on the car body acceleration is investigated.

\section{Vehicle-track interaction model}

The vehicle-track interaction model is divided into two sub-systems, namely the vehicle and the ballasted track sub-system, as shown in Figure 1. For the sake of simplicity, the track is approached by DSM in two dimensions and only vertical vibrations are considered. Additional assumptions are listed as:

(1) The vehicle runs over the ballasted track at constant speed $v$.

(2) The vehicle is composed of one car body, two bogies, four wheel sets, and a number of linear spring-dashpot units connecting the components and representing the primary and secondary suspensions.

(3) The car body is modeled as an Euler-Bernoulli beam with free ends, supported on the secondary suspension.

(4) The other components of the vehicle, i.e. the bogie and wheel sets, are assumed rigid.

(5) A typical ballasted track is selected and modelled by DSM.

(6) The wheel/rail contact force is represented by a linearized Hertzian spring.

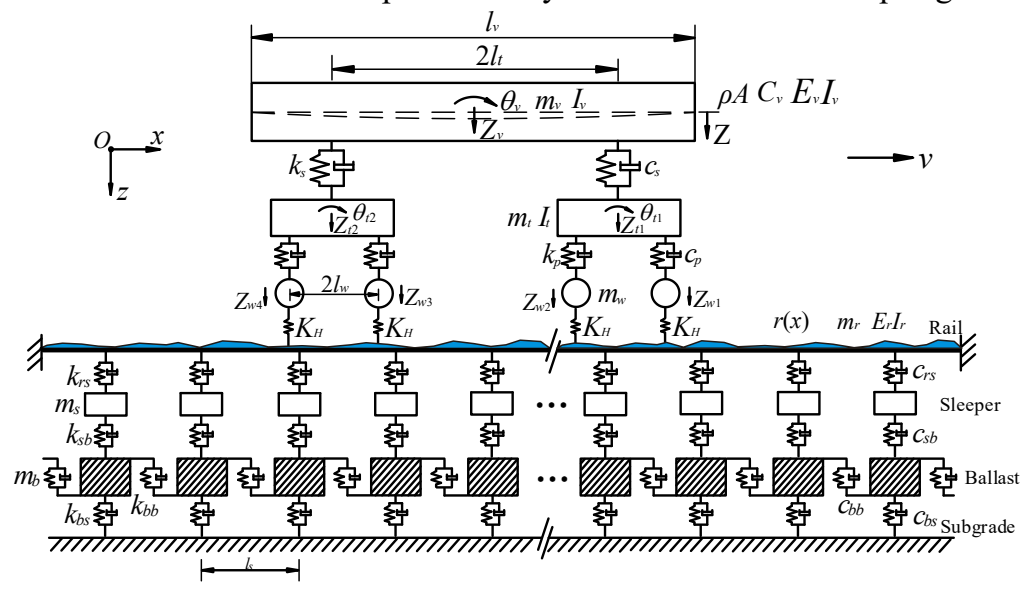

Figure 1. Vehicle-track system with flexible car-body.

\subsection{Vehicle model}

As shown in Figure 1, the car-body is modeled as a uniform Euler-Bernoulli beam supported on the secondary suspension. Its vibration can be divided into the rigid vibration, i.e. the bounce 
and pitch motion, and the flexible vibration [6]. As each bogie is assumed rigid, then has only the bounce and pitch motion. Each wheel set has only one degree of freedom vibration. To establish the equations of motion for the car body, bogies and wheel sets, the vibrations of each component are measured from their static equilibrium position. The following symbols are used for vehicle in Figure 1: $m_{v}, m_{t}$ and $m_{w}$ are the mass of the car body, bogie and wheel set, respectively. $I_{v}$ and $I_{t}$ are the pitch inertia of the car body and bogie, $E_{v} I_{v}$ stands for the flexural stiffness of the car body, $C_{v}$ and $\rho A$ are the damping coefficient and mass per unit length of the car body. Further, $l_{v}$ is the total length of the car body, $l_{t}$ and $l_{w}$ are halves of the distance between the bogie centers and the wheels set centers, respectively. $k_{s}$ and $k_{p}$ are stiffnesses, and $c_{s}$ and $c_{v}$ are viscous damping coefficients of the secondary and primary suspension system, respectively.

When the vehicle runs over the ballasted track, the equation of motion for the flexible car body can be written as

$$
\begin{gathered}
E_{v} I_{v} \frac{\partial^{4} Z(x, t)}{\partial x^{4}}+\rho A \frac{\partial^{2} Z(x, t)}{\partial t^{2}}+C_{v} \frac{\partial Z(x, t)}{\partial t} \\
=-\sum_{j=1}^{2}\left\{k_{s}\left[Z\left(l_{j}, t\right)-Z_{t j}(t)\right]+c_{s}\left[\dot{Z}\left(l_{j}, t\right)-\dot{Z}_{t j}(t)\right]\right\} \delta\left(x-l_{j}\right)
\end{gathered}
$$

$x$ is limited to $0 \leq x \leq l_{v}, l_{j}=l_{v} / 2+(-1)^{j+1} l_{t}, j=1,2$, corresponding to the front and rear bogie and $\delta$ is the Dirac delta function. $Z(x, t)$ are the vertical displacements of the car body at position $x$ and time $t$, and $Z_{t j}(t)$ is the vertical displacement of the bogie center. The flexible car body displacement can be expressed using modal superposition method as

$$
Z(x, t)=Z_{v}(t)+\left(x-\frac{l_{v}}{2}\right) \theta_{v}(t)+\sum_{n=3}^{N M_{v}} q_{n}(t) \phi_{n}(x)
$$

where $Z_{v}(t)$ and $\theta_{v}(t)$ are the vertical displacement and rotation at the car body center, describing the rigid vibration modes, $q_{n}(t)$ is the $n$-th generalized coordinate and $\phi_{n}(x)$ is the $n$-th mode shape of the flexible car body with free ends $(n>2)$.

Substituting Eq. (2) into Eq. (1) and multiplying it by $\phi_{i}(x)$ and integrating over the vehicle length, the equations of motion in modal space are given by

$$
\begin{gathered}
m_{v} \ddot{Z}_{v}(t)+k_{s} \sum_{j=1}^{2}\left[Z\left(l_{j}, t\right)-Z_{t j}(t)\right]+c_{s} \sum_{j=1}^{2}\left[\dot{Z}\left(l_{j}, t\right)-\dot{Z}_{t j}(t)\right]=0 \\
I_{v} \ddot{\theta}_{v}(t)+c_{s} l_{t} \sum_{j=1}^{2}(-1)^{j+1}\left[\dot{Z}\left(l_{j}, t\right)-\dot{Z}_{t j}(t)\right]+k_{s} l_{t} \sum_{j=1}^{2}(-1)^{j+1}\left[Z\left(l_{j}, t\right)-Z_{t j}(t)\right]=0 \\
m_{v} \ddot{q}_{i}(t)+2 \xi_{v i} \omega_{v i} m_{v} \dot{q}_{i}(t)+m_{v} \omega_{v i}^{2} q_{i}(t) \\
=-k_{s} \sum_{j=1}^{2} \phi_{i}\left(l_{j}\right)\left[Z\left(l_{j}, t\right)-Z_{t j}(t)\right]-c_{s} \sum_{j=1}^{2} \phi_{i}\left(l_{j}\right)\left[\dot{Z}\left(l_{j}, t\right)-\dot{Z}_{t j}(t)\right] i=3 \cdots N M_{v}
\end{gathered}
$$

where $\omega_{v i}$ and $\xi_{v i}$ are the $i$-th natural frequency and damping ratio of the car body.

The equation of bounce and pitch motions for the front and rear bogie read as 


$$
\begin{gathered}
m_{t} \ddot{Z}_{t j}(t)-k_{s}\left[Z\left(l_{j}, t\right)-Z_{t j}(t)\right]-c_{s}\left[\dot{Z}\left(l_{j}, t\right)-\dot{Z}_{t j}(t)\right]+\sum_{s=2 j-1}^{2 j} P_{w s}=0 \\
I_{t} \ddot{\theta}_{t j}(t)+l_{w} \sum_{s=2 j-1}^{2 j}(-1)^{s+1} P_{w s}=0
\end{gathered}
$$

where $Z_{t}(t)$ and $\theta_{t}(t)$ are the vertical displacement and rotation of the front and rear bogie, respectively, $s=1,2,3,4$ stands for the $s$-th wheel set, (when $j=1$ then $s=1$ and 2, for $j=2, s=3$ and 4), $P_{w s}$ is the force from the wheel set acting on the bogie,

$$
P_{w s}=k_{p}\left[Z_{t j}(t)+(-1)^{s+1} l_{w} \theta_{t j}(t)-Z_{w s}(t)\right]+c_{p}\left[\dot{Z}_{t j}(t)+(-1)^{s+1} l_{w} \dot{\theta}_{t j}(t)-\dot{Z}_{w s}(t)\right]
$$

where $Z_{w s}(t)$ is the vertical displacement of the $s$-th wheel set.

The equation of bounce motion for the $s$-th wheel set can be written as

$$
m_{w} \ddot{Z}_{w s}(t)+F_{w s}-P_{w s}=0
$$

where $F_{w s}(t)$ is the linear contact force for the $s$-th wheel set,

$$
F_{w s}=K_{H}\left[Z_{w s}(t)-u\left(x_{w s}, t\right)-r\left(x_{w s}\right)\right]
$$

where $u\left(x_{w s}, t\right)$ and $r\left(x_{w s}\right)$ are the rail displacement and track irregularity at location of the $s$ th wheel set. $K_{H}$ represents the contact stiffness of the linearized spring, [7]

$$
K_{H}=3 P_{0}^{1 / 3} / G
$$

where $P_{0}$ is the static axle load of the vehicle and $G$ is the contact constant.

\subsection{Track model}

As already mentioned, only the vertical vibrations of the track are considered. The typical ballasted track is modelled as DSM, i.e. as two-layer lumped mechanical model connected on top by Euler-Bernoulli beam representing the rail. The following symbols are used for the ballasted track in Figure 1: $m_{r}, m_{s}$ and $m_{b}$ are the rail mass per unit length, half sleeper and ballast mass, respectively. $E_{r} I_{r}$ is the bending stiffness of the rail, $l_{s}$ stands for the sleeper spacing and $k_{r s}, k_{s b}, k_{b s}, c_{r s}, c_{s b}, c_{b s}$ are the linear stiffness and viscous damping coefficients of the rail pad, ballast and subgrade, respectively, and $k_{b b}$ and $c_{b b}$ are the shear stiffness and viscous damping coefficient of the ballast.

The ballasted track is modelled by finite element method. Rail element length is set equal to sleepers' spacing. For the rail, the typical beam element with two nodes and four degrees of freedom is used: two vertical displacements are designated as $u_{j}$ and $u_{j+1}$ and rotations $\theta_{j}$ and $\theta_{j+1}$. For the sleeper and ballast mass vertical displacements notations $u_{s k}$ and $u_{b k}$ are used. The equation of motion of the ballasted track under moving vehicle can be written in matrix form as

$$
\left[\mathbf{M}_{T}\right]\left\{\ddot{\mathbf{U}}_{T}\right\}+\left[\mathbf{C}_{T}\right]\left\{\dot{\mathbf{U}}_{T}\right\}+\left[\mathbf{K}_{T}\right]\left\{\mathbf{U}_{T}\right\}=\left\{\mathbf{F}_{T}\right\}
$$

where $\left[\mathbf{M}_{T}\right],\left[\mathbf{C}_{T}\right]$ and $\left[\mathbf{K}_{T}\right]$ denote the mass, damping and stiffness matrices of the track system, respectively, $\left\{\ddot{\mathbf{U}}_{T}\right\},\left\{\dot{\mathbf{U}}_{T}\right\}$ and $\left\{\mathbf{U}_{T}\right\}$ are the nodal accelerations, velocities and 
displacements of the ballasted track model, respectively and $\left\{\mathbf{F}_{T}\right\}$ is the external force vector acting on the track system, that is the combination of linearized contact forces and the vehicle gravity.

\section{Solution}

The vehicle model and the track model are linked together by the linearized contact forces described by Eq. (10), thus the entire vehicle-track system is linear. Subsequently, combining the equations of motion of the vehicle and track together, all unknowns can be grouped into a vector $\left(Z_{v}, \theta_{v}, q_{n}, Z_{t j}, \theta_{t j}, Z_{w s}, u_{j}, \theta_{j}, u_{s k}, u_{b k}\right)$ and the final system in a matrix reads

$$
[\mathbf{M}]\{\ddot{\mathbf{U}}\}+[\mathbf{C}]\{\dot{\mathbf{U}}\}+[\mathbf{K}]\{\mathbf{U}\}=\{\mathbf{F}\}
$$

where $[\mathbf{M}],[\mathbf{C}]$ and $[\mathbf{K}]$ denote the mass, damping and stiffness matrices, respectively, $\{\ddot{\mathbf{U}}\},\{\dot{\mathbf{U}}\}$ and $\{\mathbf{U}\}$ denote the accelerations, velocities and displacements respectively and $\{\mathbf{F}\}$ is the external force vector acting on the vehicle-track system. To compute both the dynamic responses of the ballasted track and the vehicle with flexible car body, the equations of motion in Eq. (13) are solved by the Newmark- $\beta$ method.

\section{Numerical investigation}

By parametric analysis it was concluded that it is sufficient to consider 20 vibration modes of the flexible car body, which is the same number as used in [6]. In the following illustrative example, a time step of $0.002 \mathrm{~s}$ and running speed of $v=50 \mathrm{~km} / \mathrm{h}$ are employed to compute the dynamic responses of the vehicle-track system.

\subsection{The difference between the flexible and rigid car body}

Two vehicle models, one with flexible car body and the other one with rigid car body are considered. Displacements of the rail and accelerations of the car body center are compared in Figure 2, respectively. Displacements were extracted from the rail point located between the two adjacent sleepers, and accelerations from the flexible car body center.

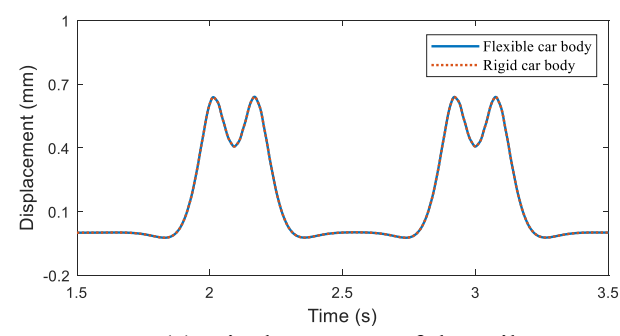

(a) Displacements of the rail

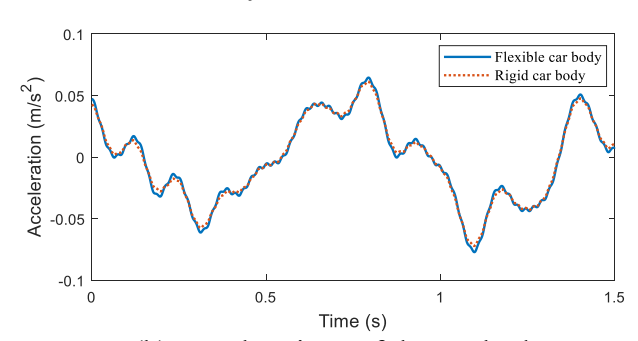

(b) Accelerations of the car body

Figure 2. Dynamic responses of the vehicle-track system.

It can be observed from Figure 2(a) that there are practically no differences between the rail displacements in these two situations. This means that the effect of the flexibility of the car body on the vibration of the ballasted track is negligible. On the other hand, as shown in Figure 2(b), the acceleration response of the car body center is larger for the flexible car body than for the rigid one. Nevertheless, this increase is quite small, and can thus be attributed to the first natural frequency of the flexible car body that is higher than for the rigid car. 


\subsection{Effect of the car body flexural stiffness}

The maximum acceleration of the car body center is plotted in Figure 3 as a function of the car body flexural stiffness. The acceleration values were extracted for discrete values of the flexural stiffness of $0.5 E_{v} I_{v}, E_{v} I_{v}, 5 E_{v} I_{v}$ and $10 E_{v} I_{v}$.

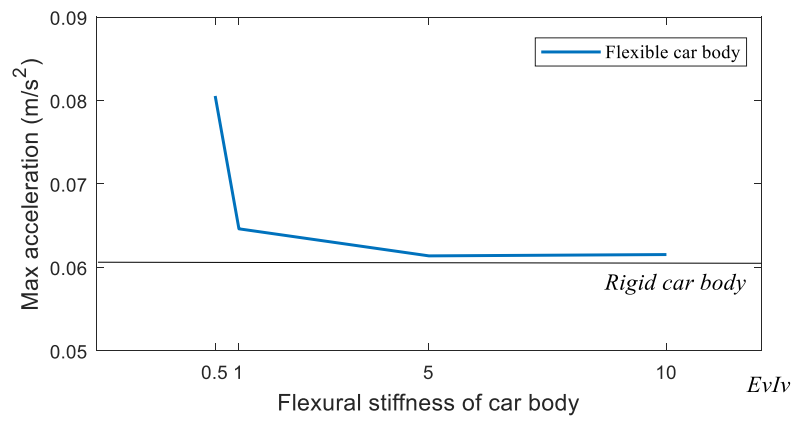

Figure 3. Maximum acceleration of the car body (blue) and the value for the rigid car body (grey).

It can be concluded from Figure 3 that with the increase of the car body flexural stiffness, the maximum acceleration at its center significantly decreases. Assuming the car body as rigid, then this value is $0.0613 \mathrm{~m} / \mathrm{s}^{2}$, which is very close to the value obtained for flexural stiffness of $5 E_{v} I_{v}$. Therefore, to improve the riding comfort of the vehicle, the flexural stiffness of the car body should be kept at a certain sufficiently high value, which is important criterion that should be respected in the lightweight design.

\section{Conclusions}

In this paper, the dynamic response of a vehicle with flexible car body moving on a ballasted track were investigated. The vehicle was modeled using flexible multibody dynamics, and the ballasted track was approached by finite element method. The following conclusions were drawn:

(1) Rail displacement is generally not affected by the flexibility of the car body. This was tested at the rail position that is located between the two adjacent sleepers.

(2) The increase of the car body flexural stiffness can lead to a significant decrease of its maximum acceleration. Therefore, the flexural stiffness of the car body should be kept at a sufficiently high value, which is important to consider in lightweight design.

This work was supported in part by the National Natural Science Foundation of China under Grant No. 51408036, the Fundamental Research Funds for the Central Universities under Grant No. 2017JBM081 and FCT, through IDMEC, under LAETA, project UID/EMS/50022/2013.

\section{References}

[1] S. Iwnicki. Handbook of railway vehicle dynamics. (CRC/Taylor \& Francis,2006)

[2] J. Evans, M. Berg. Vehicle Syst Dyn, 47, 1023-1048 (2009)

[3] K. Nguyen, J.M. Goicolea, F. Galbadon. P I Mech Eng F-J Rai, 228, 128-142 (2014)

[4] A.F.S. Rodrigues, Viability and applicability of simplified models for dynamic analysis of railway tracks, Ph.D. thesis, Nova University of Lisbon, Portugal (2017)

[5] J.N. Varandas, A. Paixao, E. Fortunato. Comput Struct, 189, 49-61 (2017)

[6] Y.J. Wang, J. Shi, Y. Xia. J Vibroeng, 14, 1824-1832 (2012)

[7] F. Lu, D. Kenned, F.W. William, J.H. Lin. J Sound Vib, 317, 236-249 (2008) 